\title{
Estudo sobre a eficácia da aerostasia pulmonar, em modelo animal, utilizando diferentes tipos de suturas*
}

\author{
Study about the ability of the pulmonary aerostasia, in animal \\ model, using differents parenchymal pulmonary types of the sutures
}

\author{
DARCY RIBEIRO PINTO FILHO ${ }^{1}$ (TE SBCT)
}

\begin{abstract}
Introdução: A busca de um modelo perfeito de aerostasia pulmonar, após cirurgias que envolvam ressecções parciais, permanece um desafio para a prática da cirurgia torácica.
\end{abstract}

O bjetivo: A valiar e comparar, em um modelo animal (suínos), a eficácia de quatro diferentes tipos de sutura pulmonar em manter aerostasia.

Método: Estudo experimental, ex vivo, em pulmões de suínos, realizado no biotério da U niversidade de Caxias do Sul. Q uatro tipos de sutura pulmonares foram avaliadas: tipo 1, sutura manual com fios cirúrgicos absorvíveis; tipo 2, grampeador exclusivo; tipo 3, grampeador recoberto por pericárdio bovino e tipo 4, grampeador recoberto por cola biológica. As suturas foram submetidas a níveis crescentes de pressão, que variaram de $10 \mathrm{cmH}_{2} \mathrm{O}$ a $60 \mathrm{cmH}_{2} \mathrm{O}$. 0 teste do borracheiro avaliou o hermetismo das suturas.

Resultados: A média de pressão em que se observou perda do hermetismo pulmonar foi de $29 \mathrm{cmH}_{2} \mathrm{O}$ no tipo 1 (n $=10) ; 38,5 \mathrm{cmH}_{2} \mathrm{O}$ no tipo $2(\mathrm{n}=10) ; 44 \mathrm{cmH}_{2} \mathrm{O}$ no tipo 3 $(n=10)$ e $51,4 \mathrm{cmH}_{2} \mathrm{O}$ no tipo $4(\mathrm{n}=10)$. A comparação entre as médias mostrou diferença estatística apenas entre as suturas tipo 1 e as suturas tipo 3 e $4, p=0,04$ e $p<$ 0,01 .

Conclusão: As suturas pulmonares com grampeadores revestidos por pericárdio bovino ou recobertos por cola biológica, quando comparadas às suturas que utilizaram fio cirúrgico isoladamente, mostraram maior eficácia em manter aerostasia pulmonar em pulmões de suínos. No entanto, não há diferença na eficácia em manter aerostasia pulmonar de suínos, quando se utilizaram suturas com grampeadores isolados ou revestidos por pericárdio bovino ou cola biológica. (J Pneumol 2003;29(5):295-301)

Descritores - Cirurgia torácica. Cola biológica. Grampeadores.

* Trabalho realizado na U niversidade de Caxias do Sul, RS.

1. Professor de Cirurgia Torácica. Doutor em Medicina - Pneumologia. Título de especialista pela Sociedade Brasileira de Cirurgia Torácica.
Background: The search for an ideal procedure to accomplish aerostasis, after partial surgical resection of the lung parenchyma, remains a practical challenge for the thoracic surgeon.

O bjective: The objective of this study was to compare the ability of four types of parenchymal pulmonary sutures in preventing air leaks, using a porcine model with incremental endobronchial pressures.

Method: Ex vivo experimental study in porcine lungs $(n=5)$ at the Laboratory of Experimental Surgery of the U niversidade de Caxias do Sul. Four different parenchymal pulmonary types of suture were analyzed: type 1 (absorbable suture), type 2: (stapled suture), type 3 (stapled suture with bovine pericardium) and type 4 (stapled suture with biologic glue). The surgical sutures $(n=40)$ were exposed to different intrabronchial pressure levels, varying from $10 \mathrm{cmH}_{2} \mathrm{O}$ to $60 \mathrm{cmH}_{2} \mathrm{O}$. The presence of air leaks along the suture line was verified through the water seal maneuver.

Results: The mean intrabronchial pressure level needed to cause suture line air leaks for each type were: type 1 ( $n=10): 29$ $\mathrm{CmH}_{2} \mathrm{O}$; type $2(\mathrm{n}=10)$ : $38.5 \mathrm{cmH}_{2} \mathrm{O}$; type $3(\mathrm{n}=10): 44 \mathrm{cmH}_{2} \mathrm{O}$; and type $4(\mathrm{n}=10): 51.4 \mathrm{cmH}_{2} \mathrm{O}$. The comparison between the mean intrabronchial pressure level of type 1 and of types 2 and 3 sutures was statistically significant, respectively: $p=0.04$ and $p=0.01$. However, the comparison between types 2,3 and 4 did not show statistic significance $(p>0.05)$.

Conclusions: The pulmonary suture covered by biologic glue demonstrated more resistance to incremental levels of intrabronchial pressure. Parenchymal pulmonary sutures using stapled suture exclusively or stapled with bovine pericardium or biologic glue demonstrated an increased ability to avoid air leaks if compared to absorbable sutures in a model of porcine lung with incremental levels of intrabronchial pressure. There were no differences between stapler exclusively or stapler and bovine pericardium or biologic glue.

Key words - Experimental study. Thoracic surgery. Biologic glue. Staplers.

Endereço para correspondência - Rua Arcy da Rocha Nóbrega, 401/ 201 - 95040-000 - Caxias do Sul, RS. Tel.: (54) 228-4882; e-mail: darcyrp@terra.com.br

Recebido para publicação em 27/5/03. Aprovado, após revisão, em 31/7/03. 


\section{INTRODUÇÃO}

Dentre os desafios transoperatórios e pós-operatórios das ressecções pulmonares parciais (lobectomia, segmentectomia ou ressecções em cunha) e, atualmente, da cirurgia redutora do volume pulmonar, estão a prevenção da fuga aérea, seja esta expressa através do coto brônquico ou do parênquima pulmonar, e a ocupação do espaço pleural pelo pulmão remanescente. Sabidamente, os índices de morbidade (infecções pleurais, sepse), tempo de internação hospitalar e mortalidade relacionados aos procedimentos que envolvem a cirurgia pulmonar aumentam, consideravelmente, nos casos de escape aéreo prolongado no pós-operatório.(1) Nos casos específicos de fístulas do coto brônquico, a utilização de suturas com fios inabsorvíveis, a manutenção de adequada irrigação sanguínea do coto brônquico e a cobertura da broncorrafia com retalhos pediculados de tecidos intratorácicos (pleura parietal, gordura pericárdica, músculos intercostais) reduziram sua incidência de $50 \%$ na década de 50 para índices que, nos dias atuais, variam de $1,6 \%$ a 6,8\%, $(2,3)$

No entanto, as fístulas broncopleurais relacionadas com o parênquima pulmonar algumas vezes são de muito difícil controle e representam o fator que motivou o desenvolvimento deste estudo.

O surgimento dos grampeadores mecânicos e sua aplicação na cirurgia pulmonar foram, sem dúvida, marco significativo na evolução da técnica operatória das ressecções pulmonares. ${ }^{(4)} \mathrm{Um}$ hermetismo mais efetivo e a diminuição do tempo operatório são avanços relacionados ao emprego sistemático dos grampeadores, principalmente na delimitação de cissuras incompletas em ressecções pulmonares parciais, na cirurgia do enfisema bolhoso e, mais recentemente, na cirurgia redutora do volume pulmonar. ${ }^{(5,6)}$

O uso exclusivo de grampeadores, ainda que melhore a efetividade da aerostasia, pode permitir algum grau de escape de ar, principalmente nos ângulos das linhas de grampos, durante a reexpansão pulmonar transoperatória. Este escape de ar é mais significativo nos pacientes portadores de enfisema pulmonar, pela inerente perda da integridade da elasticidade pulmonar. ${ }^{(7)}$ Estas observações embasaram a indicação da cobertura da linha de grampos por algum material que determinasse maior hermetismo da sutura. Os melhores resultados foram obtidos com o uso de pericárdio bovino e cola biológica. ${ }^{(8-11)}$

$\mathrm{Na}$ tentativa de solucionar esse problema, surgiram artigos $^{(6-12)}$ mostrando a utilização de grampeadores recobertos por materiais como o pericárdio bovino ou a cola de fibrina, a inversão das paredes das bolhas enfisematosas e muitos outros.

A retomada, por parte do grupo de Cooper, ${ }^{(6)}$ da proposta de Brantigan ${ }^{(13)} \mathrm{em}$ reduzir o volume pulmonar dos

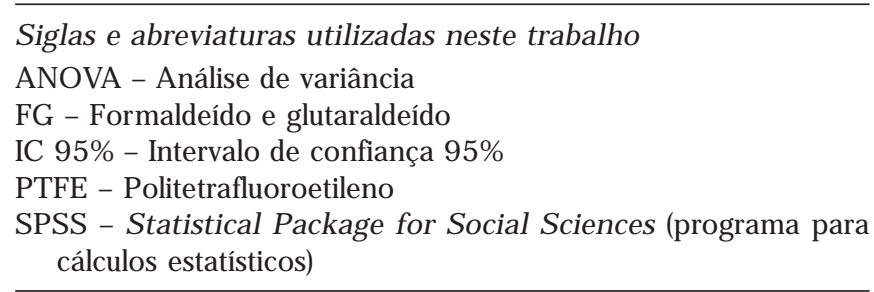

pacientes enfisematosos e permitir melhor desempenho do diafragma, trouxe o desafio de operarmos pulmões extremamente comprometidos pela doença enfisematosa e a peculiar dificuldade em mantermos o hermetismo da sutura pulmonar num tecido quase totalmente desprovido de sustentação elástica. 0 próprio $\mathrm{Cooper}^{\left({ }^{(7)} \mathrm{em} \text { uma }\right.}$ população de pacientes enfisematosos submetidos à cirurgia redutora do volume pulmonar, mostrou a importância da cobertura da linha de sutura do grampeador com pericárdio bovino, o que, segundo suas observações, eliminava virtualmente o escape de ar no pós-operatório.

A revisão da literatura sobre 0 assunto demonstra resultados heterogêneos quanto à capacidade dos diversos métodos propostos em aumentar a eficácia da aerostasia pulmonar, e não apresenta estudos comparativos.

$\mathrm{Na}$ busca de um modelo que tornasse mais eficaz a prevenção do escape de ar pós-operatório em cirurgias que envolvam o parênquima pulmonar, testamos a aerostasia de quatro tipos de sutura pulmonar, em suínos, submetidos a níveis pressóricos intrabrônquicos crescentes com observação do escape aéreo pela linha de sutura ao teste de imersão** ("borracheiro").

O modelo experimental avaliou e comparou, ex vivo, a eficácia da aerostasia pulmonar em suínos, através da sutura pulmonar manual com fio absorvível, sutura pulmonar com grampeador exclusivo, sutura pulmonar com grampeador recoberto por pericárdio bovino e sutura pulmonar com grampeador recoberto por cola biológica.

\section{MÉTOdo}

Q uarenta suturas pulmonares foram realizadas em cinco animais (suínos), comparando quatro tipos de sutura pulmonar e sua eficácia em manter o hermetismo (aerostasia), quando submetidas a níveis crescentes de pressão intrabrônquica ao teste da submersão** ("borracheiro").

0 tempo médio transcorrido entre a retirada do bloco pulmonar, a realização das suturas e o teste pressórico foi de 45 minutos.

0 estudo foi realizado no Biotério da Universidade de Caxias do Sul-RS. Os quatro diferentes tipos de suturas pulmonares, ex vivo, foram realizadas em cada pulmão do suíno, de forma rodiziada. 
Os animais foram tratados de acordo com as normas do Guide for care and use of laboratory animals (NIH número 80-23, 1978, revisão 1985).

\section{Tipos de sutura}

Sutura tipo 1: Sutura aerostática contínua e hemostática com pontos separados em "U" distantes $5 \mathrm{~mm}$ um do outro com fios absorvíveis (categute cromado 0000), numa extensão de $6 \mathrm{~cm}$. Sutura tipo 2: Grampeador linear cortante exclusivo (A uto Suture International, Inc. ${ }^{\circledR}$-J omhédica-Porto A legre-Brasil) numa extensão de $6 \mathrm{~cm}$. Sutura tipo 3: Grampeador linear cortante $6 \mathrm{~cm}$, recoberto por pericárdio bovino e confeccionado pelo cirurgião no momento do experimento. (Biopatch-enxerto biológico de pericárdio bovino - Biomedical Equipamentos e Produtos Médico-cirúrgicos Ltda. São Paulo). Sutura tipo 4: Grampeador linear cortante $6 \mathrm{~cm}$, recoberto por $1 \mathrm{ml}$ de adesivo selante de fibrina aspergido sobre a linha de sutura (Beriplast ${ }^{\circledR}$ P-Centeon FarmacêuticaSão Paulo-Brasil).

\section{Experimento}

Realização dos quatro tipos de sutura pulmonar (cada lado) em locais previamente identificados: apical, médio superior, médio inferior e basal, de maneira que as suturas fossem distribuídas de forma rodiziada em ambos os pulmões.

A pós o término de todas as suturas, iniciou-se a insuflação pulmonar unilateral com pressões positivas crescentes de cinco em cinco $\mathrm{CmH}_{2} \mathrm{O}$, verificadas no manômetro do aparelho de ventilação. 0 limite inferior estabelecido foi de $10 \mathrm{CmH}_{2} \mathrm{O}$ e o limite superior de $60 \mathrm{cmH}_{2} \mathrm{O}$. O brônquio principal contralateral foi pinçado durante a insuflação.

No final de cada limite de insuflação preestabelecido, realizou-se uma pausa inspiratória sustentada de 10 segundos para que se observasse escape de ar pelas suturas.

Foi considerado como teste positivo a observação de qualquer escape de ar pela sutura ao teste de hermetismo. Foi considerado o menor nível pressórico imposto à via aérea que tenha determinado qualquer escape aéreo pela sutura.

Considerou-se teste negativo a ausência de fuga aérea até 0 limite pressórico máximo preestabelecido $\left(60 \mathrm{CmH}_{2} \mathrm{O}\right)$.

0 hermetismo (aerostasia) foi definido como ausência de qualquer fuga aérea pelas suturas, ao teste do borracheiro, quando imposta pressão positiva crescente na via aérea.

O "teste do borracheiro" é definido como submersão da sutura pulmonar em solução salina isotônica com o intuito de observar escape de ar ao ser aplicada uma pressão intrabrônquica positiva sustentada por 10 segundos.

\section{Análise estatística}

Para a comparação das pressões intrabrônquicas que pudessem produzir escape aéreo nos quatro tipos de sutura, com distribuição gaussiana dos dados, foi utilizado o teste ANOVA, e considerou-se resultado estatisticamente significante quando $p<0,05$. Para diferenciação entre os grupos foi utilizado o teste de Tukey. 0 tamanho da amostra foi calculado para alcançar um erro alfa de 0,05 e um erro beta de 0,2 .

Os dados foram processados com o auxílio do programa SPSS v 8.0.

\section{Resultados}

Considerando-se os quatro tipos de sutura testados, a média de pressão intrabrônquica em que se observou perda do hermetismo foi de $40,75 \mathrm{cmH} 20$, com desvio padrão de 14,08 e IC $95 \%$ 36,25-45,25. Os resultados das médias de ruptura do hermetismo dos diferentes tipos de sutura experimentados são mostrados na Tabela 1.

0 cálculo das diferenças das médias de pressão em que se verificou a perda do hermetismo em cada tipo de sutura mostrou que a significância estatística, através do teste de Tukey, somente foi observada entre os tipos 1 e 3, e 1 e 4 para $p=0,04$ e $p=0,01$, respectivamente. Não houve significância entre os tipos de sutura 2,3 e 4, assim como entre as suturas 1 e 2 (Tabela 2).

U ma observação dos números da Tabela 1 aponta para uma tendência de maior resistência da sutura que utilizou grampeadores recobertos por pericárdio bovino e cola biológica, o que é demonstrado na Figura 1.

\section{DISCUSSÃO}

A análise dos resultados observados no modelo experimental confirma a menor resistência da sutura pulmonar manual com fios absorvíveis, corroborando sua pouca utilização, nos dias atuais, em cirurgias de ressecções pulmonares parciais ou cirurgias que envolvam ressecção de bolha de enfisema ou redução do volume pulmonar.

TABELA 1

Médias de pressão em que ocorreu ruptura da sutura nos quatro tipos testados

\begin{tabular}{clc}
\hline Tipo de sutura & \multicolumn{1}{c}{$\begin{array}{c}\text { Médias } \\
\text { pressão }\end{array}$} & $\begin{array}{c}\text { Intervalo de confiança } \\
\mathbf{9 5 \%}\end{array}$ \\
\hline Tipo 1 & $29 \pm \pm 8,43$ & $22,97-35,03$ \\
Tipo 2 & $38,50 \pm 13,34$ & $28,95-48,05$ \\
Tipo 3 & $44 \pm 13,08$ & $34,64-53,36$ \\
Tipo 4 & $51,50 \pm 11,80$ & $43,06-59,94$ \\
Total & $40,75 \pm 14,08$ & $36,25-45,25$ \\
\hline
\end{tabular}


TABELA 2

Resultados da análise estatística (teste de Tukey) do estudo comparativo da eficácia de quatro tipos de sutura pulmonar em manter aerostasia pulmonar em modelo animal

\begin{tabular}{lrrrr}
\hline \multirow{2}{*}{ Variável } & Variável & P & \multicolumn{2}{c}{ IC 95\% } \\
\cline { 5 - 6 } & & & Inferior & Superior \\
\hline \multirow{2}{*}{ Tipo de sutura 1 } & Tipo de sutura 2 & 0,292 & $-23,74$ & 4,74 \\
& 3 & 0,036 & $-29,24$ & $-0,76$ \\
& 4 & 0,001 & $-36,4$ & $-8,26$ \\
\hline Tipo de sutura 2 & Tipo de sutura 1 & 0,292 & $-4,74$ & 23,74 \\
& 3 & 0,727 & $-19,74$ & 8,74 \\
& 4 & 0,084 & $-27,24$ & 1,24 \\
\hline Tipo de sutura 3 & Tipo de sutura 1 & 0,036 & 0,76 & 29,24 \\
& 2 & 0,727 & $-8,74$ & 19,74 \\
& 4 & 0,497 & $-21,74$ & 6,74 \\
\hline Tipo de sutura 4 & Tipo de sutura 1 & 0,001 & 8,26 & 36,74 \\
& 2 & 0,084 & $-1,24$ & 27,24 \\
& 3 & 0,497 & $-6,74$ & 21,74 \\
\hline
\end{tabular}

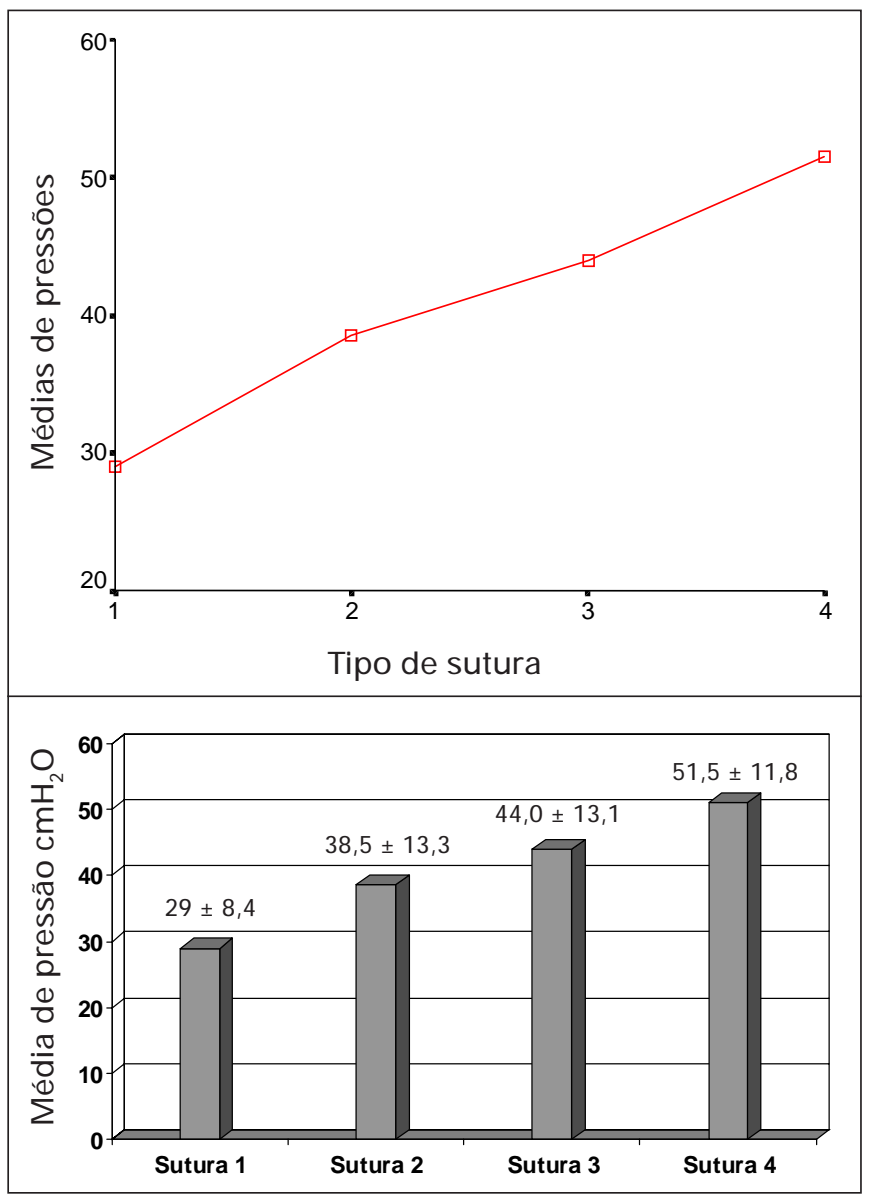

Figura 1 - Tipo de sutura e a média das pressões em que ocorreu perda da aerostasia pulmonar

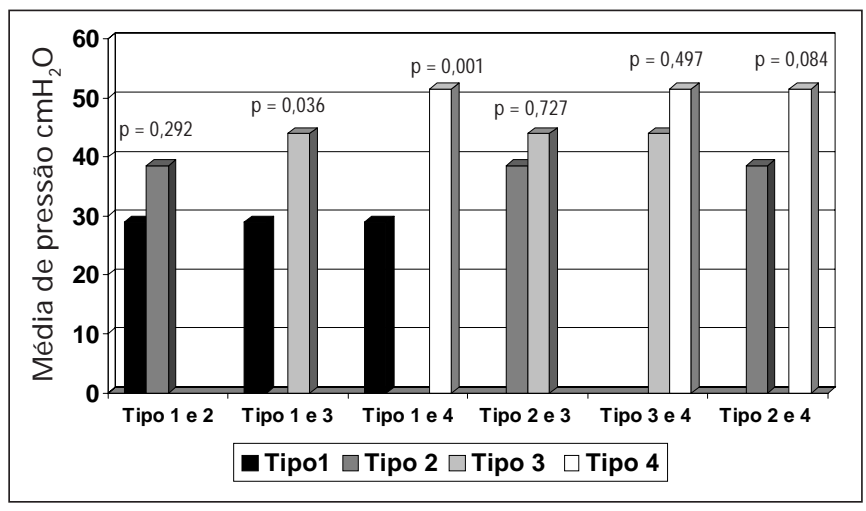

Figura 2 - Análise estatística comparando a eficácia dos quatro tipos de sutura em manter aerostasia pulmonar (teste de Tukey)

Contrariamente, o emprego de grampeadores para estas mesmas cirurgias tornou-se grandemente difundido e integrado à rotina da maioria dos serviços de cirurgia torácica no mundo inteiro. $(2,4,14)$

Miller ${ }^{(17)}$ comparou a eficácia do grampeador revestido ou não por pericárdio bovino, em 80 pacientes submetidos à ressecção pulmonar, excluindo a cirurgia redutora do volume pulmonar, e concluiu que o tempo de escape de ar no pós-operatório e o tempo de permanência dos drenos pleurais nos dois grupos não apresentaram diferença estatística significativa $(p>0,05)$.

Venuta et al. (18) e Vaughn et al.,(19) testando o uso de pericárdio bovino e politetrafluoroetileno (PTFE) para reforço da sutura pulmonar, confirmaram a eficácia do método para prevenção de escape aéreo no pós-operatório, 
quando comparado ao uso de eletrocautério e fio absorvível $(p<0,01)$.

$J$ atene et al. ${ }^{(20)}$ utilizaram grampeadores mecânicos em 150 pacientes submetidos à sutura brônquica, de parênquima pulmonar e vascular, totalizando 186 procedimentos. Estes pacientes, que eram portadores de neoplasias, doenças supurativas e malformações congênitas, não apresentaram nenhuma fístula brônquica após a sutura mecânica.

O pressuposto teórico de que a cobertura da linha de grampos com os materiais disponíveis poderia aumentar a efetividade da aerostasia pulmonar parece razoável. 0 modelo experimental testado, quando comparou os níveis de resistência das suturas que utilizaram grampeador exclusivo e aquelas recobertas por pericárdio bovino ou cola biológica, não mostrou diferença estatística significante ao serem submetidas a níveis crescentes de pressão endobrônquica $(p>0,05)$.

A aplicação tópica da cola de fibrina ou cola biológica, para reduzir escape aéreo no pós-operatório de cirurgia pulmonar, tem sido utilizada desde a década de 70, principalmente nos países europeus. Os trabalhos de Thetter ${ }^{(21)}$ e Turk et al. (22) foram os primeiros a atestarem uma melhor efetividade no controle do escape aéreo das suturas pulmonares com a utilização da cola. Thetter descreveu 0 uso de cola durante a toracotomia, tanto isolada como em combinação com pleurodese abrasiva, como método de alcançar pleurodese em pacientes portadores de pneumotórax espontâneo. Ele comparou esse grupo de pacientes com controles históricos que não usaram cola e concluiu que o tempo médio de permanência dos drenos pleurais foi menor no grupo tratado. 0 mesmo autor não observou diferenças significativas no tempo de permanência dos drenos pleurais em pacientes submetidos à ressecção pulmonar que tiveram o parênquima revestido por cola de fibrina, quando comparados a um grupo de controle histórico que não recebeu aplicação do selante. Os trabalhos de Thurk et al. ${ }^{(22)}$ foram baseados em estudos experimentais em ratos. Após submeterem dois grupos de ratos a secção traumática do parênquima pulmonar, observaram que a cobertura da lesão com cola de fibrina conferia maior resistência à pressão na via aérea quando comparada ao grupo sem revestimento por selante. A plicando esse mesmo modelo em pacientes submetidos à ressecção pulmonar, e testados no período transoperatório quanto à tolerância da sutura à pressão positiva na via aérea, observaram que nos pacientes que tiveram sua linha de sutura recoberta por cola biológica a resistência da sutura foi $36 \%$ superior à realizada no grupo que não utilizou o reforço com cola biológica $(p<0,05)$.

Nomori e Horio(23) mostraram a possibilidade de extrapolação dos resultados de cirurgias experimentais para cirurgias em humanos. 0 modelo utilizou pulmões de suí- nos e suturas pulmonares com grampeadores revestidos por formaldeído e glutaraldeído, submetidas a níveis crescentes de pressão, até atingirem o limite máximo de $60 \mathrm{cmH}_{2} \mathrm{O}$. Neste limite pressórico não se observou escape de ar pela linha de sutura. A aplicação deste mesmo modelo em pulmões de dez pacientes enfisematosos confirmou o efetivo hermetismo da sutura pulmonar com grampeador revestido por formaldeído e glutaraldeído.

N um artigo clássico, Mouritzen et al.(24) avaliaram 114 pacientes submetidos a ressecções pulmonares, estratificados por lobectomia e pneumonectomia. No grupo de pacientes submetidos à lobectomia $(n=63)$ com reforço da sutura do parênquima com cola biológica (Beriplast ${ }^{\circledR}$ ), a resistência da sutura, caracterizada pela manutenção de seu hermetismo, foi observada em $67,7 \%$ dos casos e em $48,4 \%$ do grupo controle ( $p<0,01$ e IC $95 \% 58 \%-98 \%$ ). Outra variável analisada neste estudo foi a proporção de escape aéreo no pós-operatório. No grupo de pacientes submetidos a lobectomia com aplicação de cola biológica, a incidência de escape aéreo foi de 38,7\% comparado a $65,6 \%$ no grupo controle $(p<0,05)$. A duração da fuga aérea, no entanto, neste mesmo grupo de pacientes submetidos à lobectomia não apresentou diferença estatística: quatro dias no grupo que utilizou reforço com cola e cinco dias no grupo controle.

A aplicabilidade clínica dos selantes foi mostrada em um estudo mais recente, conduzido por Wong et al.,(25) que avaliaram o efeito da cola de fibrina na redução do escape aéreo alveolar pós-toracotomia. Sessenta e seis pacientes submetidos a lobectomias, segmentectomias ou descorticação pulmonar que, no transoperatório, mostrassem persistência de moderado a grave escape de ar, mesmo com a instituição das medidas convencionais de controle desta situação, foram divididos em dois grupos de 33 pacientes e estratificados pelo reforço da sutura com cola biológica. A média de permanência dos drenos pleurais e do período de internação hospitalar foi de seis e oito dias no grupo que usou cola biológica e de seis e nove dias no grupo controle. 0 autor concluiu que não houve vantagem na utilização da cola biológica em adição aos métodos convencionais de controle de escape aéreo e mencionou o custo financeiro do emprego do selante de fibrina.

A possibilidade de utilização de outras opções de selantes foi mostrada em vários trabalhos da literatura. (26-29) Kjaergard et al., ${ }^{(30)} \mathrm{em}$ um modelo semelhante ao desenvolvido em nosso estudo, submeteram um grupo de suínos a suturas pulmonares com aplicação de fibrina autóloga (Vivostat-ConvaTec; Skillman NJ ) e usaram um grupo controle em que as suturas pulmonares foram recobertas por albumina humana a $20 \%$. Sob pressão positiva crescente na via aérea, testou-se a resistência de cada material ao "teste do borracheiro". A fibrina autóloga mos- 
trou-se mais efetiva em prevenir escape aéreo $(p<0,01)$. A possibilidade de utilização de material autólogo representa um futuro promissor para os selantes. Hepatite B, hipotensão, anafilaxia, hemorragia e infecção, complicações atribuídas à cola biológica de fibrina, poderiam ser evitadas pela utilização de fibrina do sangue do próprio paciente.

A utilização de cola de fibrina em tecido pulmonar é um ponto a ser discutido. As características peculiares do pulmão, um órgão com espaços aéreos e grande quantidade de sangue em seu interior e que altera seu volume a cada movimento respiratório, torna a aplicação e a adesão de fibrina em sua superfície um desafio. Durante a polimerização do selante, dois tipos de ligações químicas formam-se dentro do adesivo: uma ligação interna do selante com o próprio selante e uma ligação externa do selante com o tecido. A ligação mais forte é a interna. Caso haja movimentação da superfície, o material pode alterar sua configuração e sua adesão tornar-se ineficaz. Nesse conceito reside, pois, a orientação técnica para a aplicação de cola biológica na superfície pulmonar. 0 local de aplicação deve estar o mais seco possível e o pulmão estabilizado por pinçamento brônquico até que se complete a reação química. (31)

Uma característica do modelo experimentado, que limita a extrapolação dos resultados, é a utilização de pulmões sadios, sem enfisema pulmonar e, portanto, com sua elasticidade preservada. U ma questão, no entanto, poderia ser formulada: poderíamos prescindir, em pacientes portadores de pulmões normais e submetidos à ressecção, de reforços da linha de sutura do grampeador? Mesmo que não tenha sido esse o objeto deste estudo, parece razoável a reflexão sobre os seus resultados, principalmente se levarmos em consideração os custos desses materiais. 0 grampeador linear cortante $6.0 \mathrm{com}$ uma carga custa, aproximadamente, $\mathrm{R} \$ 1.500,00$ e a cola biológica (Beriplast), aproximadamente $\mathrm{R} \$ 300,00$ por ml.

0 desenvolvimento de experimentos que induzam 0 surgimento de enfisema pulmonar em animais de laboratório, para que se possa testar esse modelo em pulmões doentes e, dessa forma, aproximar seus resultados de uma situação que espelhe ou represente as características da população de pacientes mais comumente submetida à cirurgia pulmonar, pode representar o seguimento desta linha de estudo experimental.

As suturas pulmonares que utilizaram grampeadores revestidos por pericárdio bovino ou foram recobertas por cola biológica, quando comparadas às suturas que utilizaram fio cirúrgico isoladamente, mostraram maior eficácia em manter aerostasia pulmonar em pulmões de suínos. No entanto, não há diferença na eficácia em manter aerostasia pulmonar de suínos, quando se utilizaram suturas com grampeadores isolados ou revestidos por pericárdio bovino ou cola biológica.

\section{REFERÊNCIAS}

1. Asamura H, Naruke T, Tsuchiya R. Bronchopleural fistulas associated with lung cancer operations. J Thorac Cardiovasc Surg 1992;104:145664.

2. Vester SR, Faber LP, Kittle CF. Bronchopleural fistula after stapled closure of bronchus. Ann Thorac Surg 1991;52:1252-7.

3. Hollaus PH, Lax F, El-Nashef BB. Natural history of bronchopleural fistula after pneumonectomy: a review of 96 cases. Ann Thorac Surg 1997;63:1391-7.

4. Amosov NM, Berezovski KK. Pulmonary resection with mechanical suture. J Thorac Cardiovasc Surg 1961;41:325-35.

5. Cooper JD. The history of surgical procedures for emphysema. Ann Thorac Surg 1997;63:312-9.

6. Cooper J D, Trulock EP. Bilateral pneumonectomy (volume reduction) for chronic obstructive pulmonary disease. J Thorac Cardiovasc Surg 1995;109:106-19.

7. Cooper J D. Technique to reduce air leaks after resection of emphysematous lung. Ann Thorac Surg 1994;57:1038-9.

8. Fischel RJ, McKenna RJ. Bovine pericardium versus bovine collagen to buttress staples for lung reduction operations. Ann Thorac Surg 1998;65:217-9.

9. McKenna RJ, Brenner M, Gelb AF, Mullin M, Singh N, Peters H. A randomized, prospective trial of stapled lung reduction versus laser bullectomy for diffuse emphysema. J Thorac Cardiovasc Surg 1996; 111:317-22

10. Fleisher AG, Evans KG, Nelems B, Finley RJ. Effect of routine fibrin glue use on the duration of air leaks after lobectomy. Ann Thorac Surg $1990 ; 49: 133-4$
11. Hillerdal G. Large emphysematous bullae - successful treatment with thoracoscopic technique using fibrin glue in poor-risk patients. Chest 1995;107:1450-3.

12. McC arthy PM, Trastek VF, Bell DG, et al. The effectiveness of fibrin glue sealant for reducing experimental pulmonary air lake. Ann Thorac Surg 1988;45:203-5.

13. Brantingan OC, Mueller E, Kress MB. A surgical approach to pulmonary emphysema. Am Rev Respir Dis 1959;80:194-202.

14. Roberson LD. A ir leaks after surgical stapling in lung resection: a comparison between stapling alone and stapling with staple-line reinforcement materials in canine model. J Thorac Cardiovasc Surg 1998;116: 353-4

15. Thistlethwaite PA, Luketich J D, Ferson PF, Keenan RJ , J amieson SW. Ablation of persistent air leaks after thoracic procedures with fibrin sealant. Ann Thorac Surg 1999;575-7.

16. Vaughn CC, Vaughn PL, Sawyer P, Manning M, Anderson D, Roseman $L$, et al. T issue response to biomaterials used for staple-line reinforcement in lung resection: a comparison between expanded polytetrafluorethylene and bovine pericardium. Eur J Cardiothorac Surg 1998; 13:259-65.

17. Miller J I, Landreneau RJ , Wright CE. A comparative study of buttressed versus nonbuttressed staple line in pulmonary resections. Ann Thorac Surg 2001;71:319-23.

18. Venuta F, Rendina EA, DeGiacomo TE. Techniques to reduce air leaks after pulmonary lobectomy. Eur J Cardiothorac Surg 1998;13:361-4.

19. Vaughn CC, Wolner E, Dahan M. Prevention of air leaks after pulmonary wedge resection. Ann Thorac Surg 1997;63:864-6. 
20. J atene FB, Pego-Fernandes PM, Galvão PM. U so de grampeadores mecânicos em ressecção broncopulmonar. J Pneumol 1996;22:291 4.

21. Thetter 0 . Fibrin adhesive and its application in thoracic surgery. J Thorac Cardiovasc Surg 1981;29:290-2.

22. Turk R, Weidringer J W, H artel W, et al. Closure of lung leaks by fibrin gluing. Experimental investigations and clinical experiences. J Thorac Cardiovasc Surg 1983;31:185-6.

23. Nomori H, Horio H. Gelatin-Resorcinol-formaldehyde-glutaraldehyde glue-spread stapler prevents air leakage from the lung. Ann Thorac Surg 1997;63:352-5.

24. Mouritzen $C$, Drömer $M, K$ einecke $H O$. The effect of fibrin glueing to seal bronchial and alveolar leakages after pulmonary resections and decortications. Eur J Cardiothorac Sur 1993;7:75-80.

25. Wong K, Goldstraw P. Effect of fibrin glue in the reduction of posthoracotomy alveolar air leak. Ann Thorac Surg 1997;64:979-81.
26. Dunn J C, Goa Karen. Fibrin sealant. A review of its use in surgery and endoscopy. Drugs 1999;58:863-86.

27. Feito $B A$, Rath $A M$, Longchampt $E$, Azorin J. Experimental study on the in vivo behavior of a new collagen glue in lung surgery. Eur Cardiothorac Surg 2000;17:8-13.

28. York LE, Lewall MD, Hirji M, Gelfand ET, Modry DL. Endoscopic diagnosis and treatment of postoperative bronchopleural fistula. Chest 1990:90:1390-2.

29. Kodama K, Doi O, H igashiyama. Pneumostatic effect of gelatin resorcinol formadehyde-glutaraldehyde glue on thermal injury of the lung: an experimental study on rats. Eur J Cardiothorac Surg 1997;11:333-7.

30. Kjaergard HK, Pedersen JH, Krasnik M, Weis-Fogh US, Fleron $\mathrm{H}$, Griffin HE. Prevention of air leakage by spraying Vivostat fibrin sealant after lung resection in pigs. Chest 2000;117:1124-7.

31. Bayfield MS, Spotnitz WD. Fibrin sealant in thoracic surgery. Chest Surg Cli N Am 1996;3:567-83. 